\title{
Stage II Esophageal Cancer AJCC v6
}

National Cancer Institute

\section{Source}

National Cancer Institute. Stage /I Esophageal Cancer A/CC v6. NCI Thesaurus. Code C9211.

Stage II includes: IIA: (T2, N0, M0); (T3, N0, MO) and IIB: (TI, NI, MO); (T2, NI, MO). TI:

Tumor invades lamina propria or submucosa. T2: Tumor invades muscularis propria. T3:

Tumor invades adventitia. N0: No regional lymph node metastasis. N1: Regional lymph node metastasis. M0: No distant metastasis. (AJCC 6th ed.) -2003 